DOE/ER/14079--23

DE93 006558

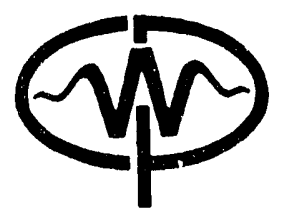

\title{
Seismic Modeling and Reverse-Time Depth Migration by Flux-Corrected Transport
}

Tong Fei

Center for Wave Phenomena

Colorado School of Mines

Golden, Colorado 80401

(303) 273-3537 


\title{
Seismic modeling and reverse-time depth migration by flux-corrected transport
}

\author{
Tong Fei
}

\begin{abstract}
Where the Earth's subsurface is generally inhomogeneous, lateral and vertical variation in velocity and density should be considered when doing seismic modeling and migration. Finite-difference modeling and reverse-time depth migration based on the full wave equation are approaches that take such variation into account.

Here, through a change of dependent variables, the second-order acoustic wave equation is replaced by four first-order partial differential equations. The fluxcorrected transport (FCT) method, commonly used in hydrodynamics for shockwave simulation, can then be used in solving these equations. The FCT method offers the opportunity to preserve a broader range of frequencies at lower computational cost than in conventional finite-difference modeling and reverse-time wave extrapolation. Moreover, it is also applicable in circumstances involving discontinuities in the wavefield, where other conventional finite-difference approaches fail. Computed two-dimensional impulse responses and synthetic data indicate that this method can accurately image positions of reflectors with greater than 90-degree dip for variable-velocity media.
\end{abstract}

\section{INTRODUCTION}

Finite-difference methods, following the approaches of Claerbout (1985), have been widely implemented for wave extrapolation in modeling and migration. Those approaches employ a one-way wave equation that allows energy to propagate either downward or upward, but not both. Although successful in many situations, such methods are limited by the assumptions made in deriving the one-way wave equation. In particular, it is assumed that the spatial derivatives of the velocity : $:$ ld can be ignored. However, such terms are significant in the presence of strong velocity contrasts. 
Moreover, most finite-difference schemes based on the one-way wave equation contain a limit on the maximum dip angle of the reflector. To deal with a variable velocity field, Kosloff and Baysal (1983) developed a two-dimensional migration scheme in the frequency and space domain based on a direct integration in depth of the acoustic wave equation.

Reverse-time migration methods based on full wave equation potentially can address all issues - arbitrary velocity variations, turning waves, shear waves and anisotropy - anything that can be treated in seismic modeling. This method, however is costly, placing heavy demands on computer memory and input/output devices (Reshef and Kessler, 1989; Blacquiere et al., 1989; Hale and Witte, 1992).

Some finite-difference schemes for numerically solving the wave equation suffer from undesirable ripples, particularly near large gradients in wavefields or when too coarse a computation grid is used. Alford et al. (1974) and Kelly et al. (1976) have studied the numerical dispersion existing in finite-difference methods. They concluded that the signals computed by finite-difference methods become strongly dispersed if the grid is too coarse. For eliminating the numerical dispersion, they state that at least 11 and 5.5 points per wavelength must be used for the secondorder and fourth-order finite-difference methods, respectively. Other schemes, such as a two-step explicit method on a staggered grid, produce no ripples, but suffer from excessive smoothing of waveforms and loss of amplitude (numerical diffusion). The flux-corrected transport (FCT) method developed by Boris and Book (1973) has been successfully applied in the solution of the continuity equation for acoustic media in circumstances involving large gradients and discontinuities, where standard algorithms fail. The FCT algorithm can treat strong gradients, shocks and impulses, without the usual dispersively generated ripples, even for five or fewer points per wavelength.

Here, the second-order accuracy, FCT finite-difference technique, is applied to forward seismic modeling and reverse-time depth migration. The method requires that the second-order acoustic wave equation first be replaced by an equivalent set of first-order partial differential equations. This method can be applied with full consideration of variable velocity and density, and can image reflectors with a wide range of dips. It can also be used on a relatively coarse grid to achieve accuracy comparable to that of conventional finite-difference approaches on a finer grid.

\section{THEORY}

The key steps in deriving equations used for FCT modeling and reverse-time depth migration here are (1) to define new dependent variables, (2) to form four irst-order, so-called conservative, equations in these variables, based on the original secondorder acoustic wave equation, and (3) apply the FCT technique to the conservative equations. 


\section{Equations}

For velocity and density fields that are functions of space, the acoustic wave equation is

$$
\frac{1}{\rho} \frac{\partial^{2} P}{\partial^{2} t}=c^{2}\left[\frac{\partial}{\partial x}\left(\frac{1}{\rho} \frac{\partial P}{\partial x}\right)+\frac{\partial}{\partial y}\left(\frac{1}{\rho} \frac{\partial P}{\partial y}\right)+\frac{\partial}{\partial z}\left(\frac{1}{\rho} \frac{\partial P}{\partial z}\right)\right]+f(x, y, z, t),
$$

where $\rho=\rho(x, y, z)$ is density, $c=c(x, y, z)$ is wave velocity, and $f(x, y, z, t)$ is the source function.

With new dependent variables defined as

$$
\begin{gathered}
q \equiv \frac{1}{\rho c^{2}} \frac{\partial P}{\partial t} \\
u \equiv \frac{\partial P}{\partial x} \\
v \equiv \frac{\partial P}{\partial y} \\
w \equiv \frac{\partial P}{\partial z}
\end{gathered}
$$

the second-order acoustic wave equation (1) is reduced to a new first-order partial differential equation that has the conservative form, and three additional first-order partial differential equations are derived from definitions (2) through (5). The four new equations give a complete set of equations in the form required for doing the wavefield extrapolation by the FCT technique. These equations take the form

$$
\begin{gathered}
\frac{\partial q}{\partial t}=\frac{\partial}{\partial x}\left(\frac{u}{\rho}\right)+\frac{\partial}{\partial y}\left(\frac{v}{\rho}\right)+\frac{\partial}{\partial y}\left(\frac{w}{\rho}\right)+f, \\
\frac{\partial u}{\partial t}=\frac{\partial}{\partial x}\left(\rho c^{2} q\right), \\
\frac{\partial v}{\partial t}=\frac{\partial}{\partial y}\left(\rho c^{2} q\right), \\
\frac{\partial w}{\partial t}=\frac{\partial}{\partial z}\left(\rho c^{2} q\right) .
\end{gathered}
$$

From equation (2), note that the new dependent variable $q(x, y, z, t)$ has a 90 degree phase shift relative to the variable $P$. 


\section{Multi-dimensional flux-corrected transport}

Conceptually, the FCT algorithm consists of two major stages - a transport stage (Stage I), followed by an anti-diffusive or corrective stage (Stage II). Interaction of these two stages enables FCT algorithms to treat strong gradients and shocks with almost none of the usual dispersively generated ripples.

In the first stage, a standard finite-difference method, such as leapfrog, is used. Stage II is devoted to correcting numerical errors introduced in Stage I.

The Appendix gives the procedure for solving equations (6) through (9) by the flux-corrected transport (FCT) finite-difference method. In the computer implementation, the absorbing boundary condition of Clayton and Engquist (1980) has been applied to the two side boundaries and the lower boundary.

With this flux-corrected transport technique, we obtain a depth migration image for spatially variable media at less computational effort (generally five times less) than in conventional finite-difference reverse-time methods, which require a finer grid and smaller time step to achieve comparable accuracy.

\section{NUMERICAL EXAMPLES}

The FCT algorithm was tested on one-dimensional forward problems and on twodimensional reverse-time depth migration. The reverse-time depth migration, which basically is the same as forward modeling, simply runs time backwards.

For the one-dimensional case, where $\frac{\partial}{\partial x}=\frac{\partial}{\partial y}=0$, forward modeling tests are made for a wavelet that is an isolated full-cycle of a sinusoid, $0.5 \cos (2 \pi f t)+0.5$, with different frequencies $f$, as well as for a rectangle function with different widths. The medium is homogeneous in this case.

For the two-dimensional case where $\frac{\partial}{\partial y}=0$, input data in one set of tests consist of unit impulses for three media, the first with a constant velocity, the second with a constant velocity gradient in depth $z$, and the third with a constant lateral velocity gradient. In other tests involving a reflector model, both constant velocity and $v(x, z)$ velocity fields are considered.

\section{One-dimensional tests}

In these one-dimensional tests, the medium has a constant density and a constant velocity of $2 \mathrm{~km} / \mathrm{s}$. The spatial step size $\Delta x$ is $0.01 \mathrm{~km}$, and the time step $\Delta t$ is $2 \mathrm{~ms}$. In each of the modeling tests, I specify a time sequence at the surface and examine a snapshot of the wavefield generated in depth. Figures 1 through 4 show the snapshots at $1 \mathrm{~s}$, generated by three isolated sinusoids at the surface 0.33-s apart. The frequencies for these tests are $10 \mathrm{~Hz}$ (twenty samples per wavelength), $15 \mathrm{~Hz}$ (thirteen samples per wavelength), $25 \mathrm{~Hz}$ (eight samples per wavelength) and $40 \mathrm{~Hz}$ (five samples per wavelength) respectively. Similarly, Figures 5 through 7 show the 
snapshots at $1 \mathrm{~s}$ for rectangle function pulses at the surface with eight, four and two samples per pulse width, respectively.

In each of the seven figures, I compare the results for the ideal solution with those obtained by a standard finite-difference method and by the FCT algorithm. The ideal solution has a waveform that is unchanged during propagation. In Figure 1, the low-frequency simulation (large dominant wavelength relative to $\Delta x$ ), the standard leapfrog method applied to first-orler equations gives a good result with only a small amount of dispersion, while the dispersion ripples are absent in the FCT solution.

For the relatively higher-frequency $(15 \mathrm{~Hz}$ ) case shown in Figure 2, the dispersive ripples generated from the standard leapfrog method are increased, but again no dispersion is evident in the FCT result. The FCT solution, however, has amplitudes that are less than 3 percent weaker than the correct solution, while the leapfrog solution has no amplitude loss. The weakened amplitudes are the result of applying diffusion and anti-diffusion in the FCT method; the anti-diffusion did not fully correct for the amplitude loss in the diffusion stage.

For the waves with short periods, eight samples per wavelength (Figure 3) and five samples per wavelength (Figure 4), the standard leapfrog method yields a progressively more distorted and weakened waveform, with large precursor oscillations. The FCT method still produces a good waveform shape, without the dispersive ripples, but with the amplitude losses of about 5 percent in Figure 3 and about 10 percent (for the deepest event) in Figure 4. The results suggest that the FCT method can give the correct position of the wavefront even when few samples per wavelength are used, but the error in amplitude seems to be unavoidable.

For the rectangle pulses (Figures 5 through 7), the standard leapfrog finitedifference method gives poor solutions for all choices of sampling, whereas the FCT solutions show little distortion in waveform and no numerical dispersion. Also for the choices of eight and four samples per pulse width, the FCT method gives the correct amplitudes (Figures 5 and 6), because relatively lower frequencies are dominant in these two cases than in tests with the sinusoidal pulses. For the narrowest rectangle pulse, with two samples per pulse width, which has wider range of frequency components than that of the previous two cases, the distorted rectangle pulse has significant loss of amplitude, as shown in Figure 7.

When the conventional second-order finite-difference method with eleven points per wavelength and fourth-order finite-difference method with six points per wavelength are used to solve wave equation (1), no dispersion is observed for both methods if the wave is propagating for a sufficiently short time (Alford et al., 1974; Kelly et al., 1976). If the wave propagates for a longer time (i.e., to a greater depth), both the second-order and the fourth-order finite-difference methods would generate strong numerical dispersion for a sinusoid with the above choices of points per wavelength (Figure 8a and Figure 9a). When using twenty points per wavelength for the secondorder method and twelve points for the fourth-order method, the numerical dispersion is much reduced (Figure $8 \mathrm{~b}$ and Figure $9 \mathrm{~b}$ ). Applying the second-order and the fourth- 


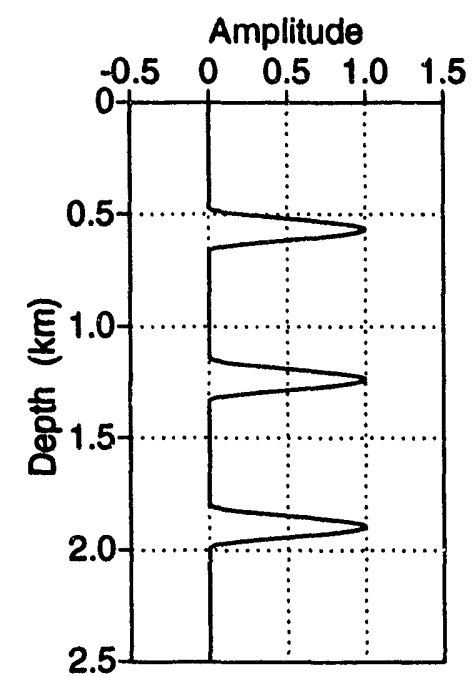

(a)

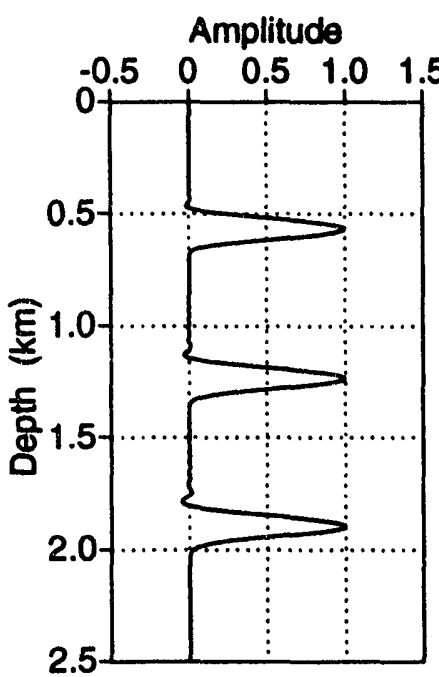

(b)

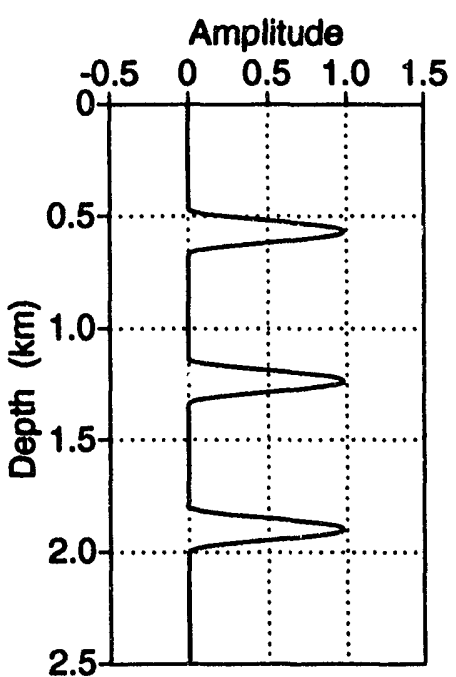

(c)

Fig. 1. One-dimensional test for isolated full-cycle of a sinusoid with a frequency of $10 \mathrm{~Hz}$ (twenty samples per wavelength), for a constant velocity of $2 \mathrm{~km} / \mathrm{s}$ and a constant density. (a) Ideal wavefield. (b) Wavefield obtained by a standard leapfrog finite-difference method. (c) Wavefield obtained with the FCT correction.

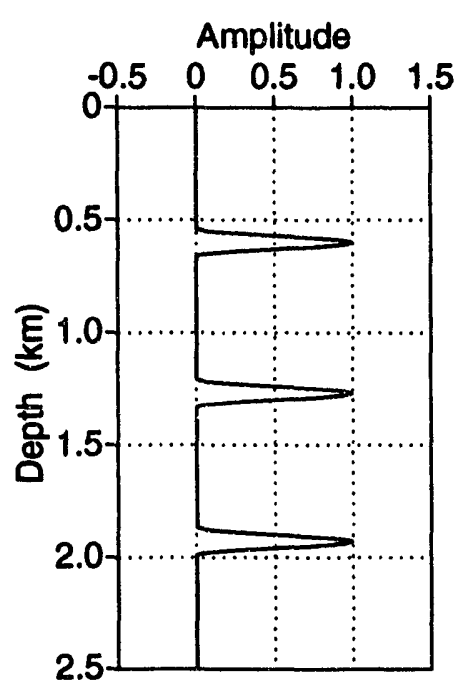

(a)

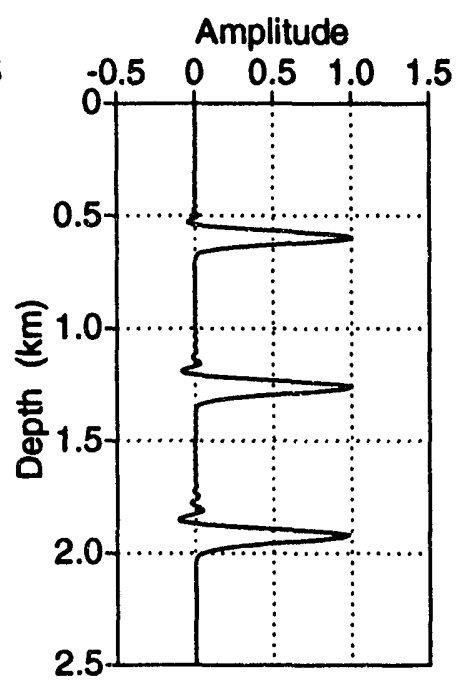

(b)

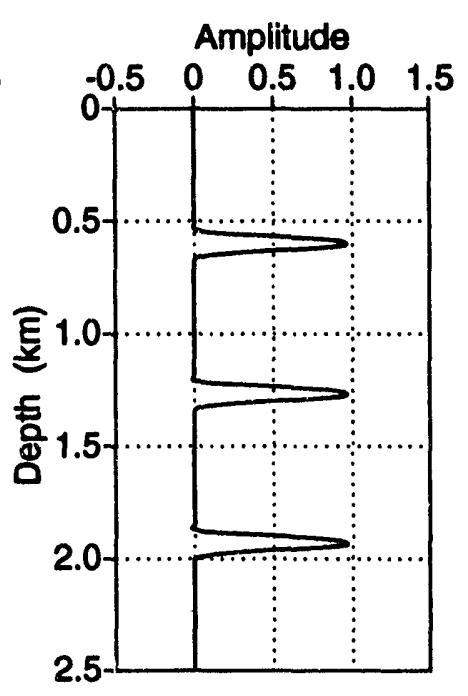

(c)

FIG. 2. One-dimensional test for isolated full-cycle of a sinusoid with a frequency of $15 \mathrm{~Hz}$ (thirteen samples per wavelength), for a constant velocity of $2 \mathrm{~km} / \mathrm{s}$ and a constant density. (a) Ideal wavefield. (b) Wavefield obtained by a standard leapfrog finite-difference method. (c) Wavefield obtained with the FCT correction. 


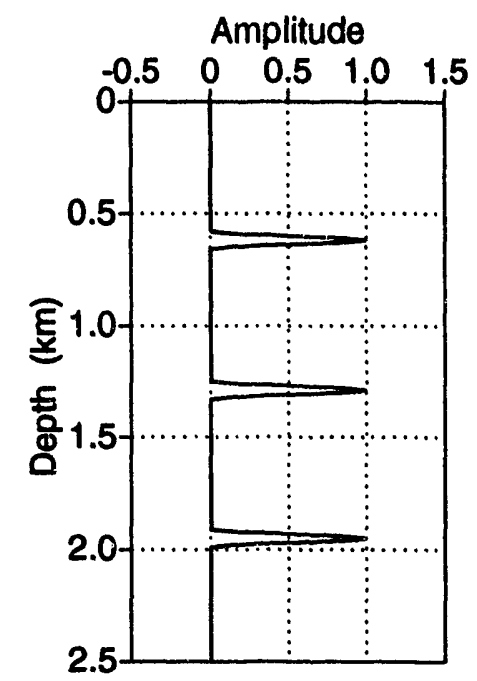

(a)

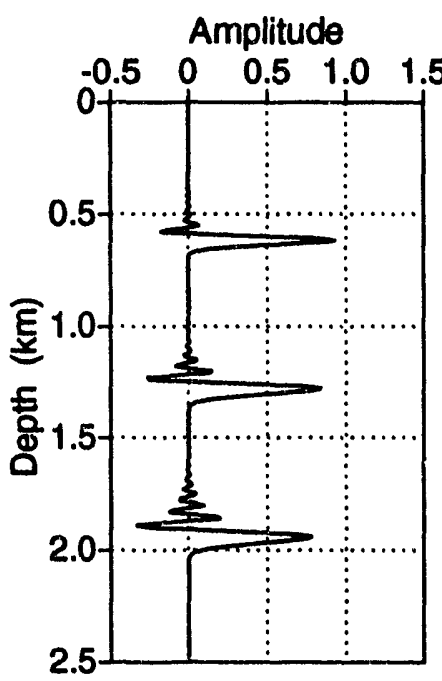

(b)

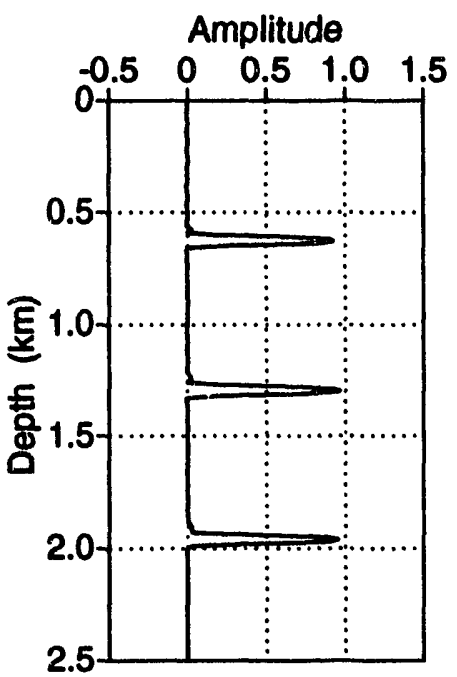

(c)

FIG. 3. One-dimensional test for isolated full-cycle of a sinusoid with a frequency of $25 \mathrm{~Hz}$ (eight samples per wavelength), for a constant velocity of $2 \mathrm{~km} / \mathrm{s}$ and a constant density. (a) Ideal wavefield. (b) Wavefield obtained by a standard leapfrog finite-difference method. (c) Wavefield obtained with the FCT correction.

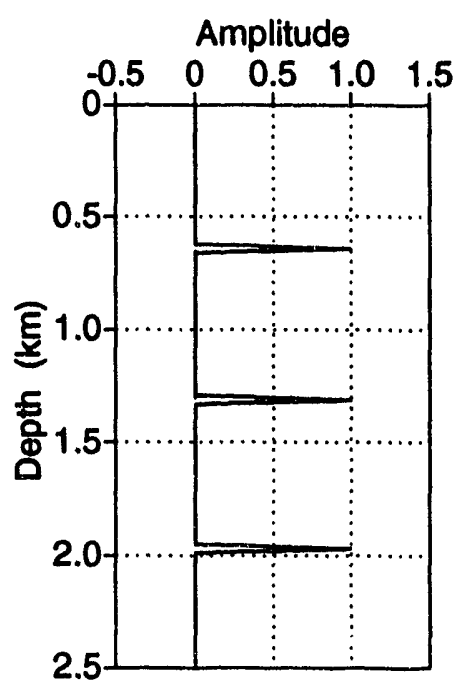

(a)

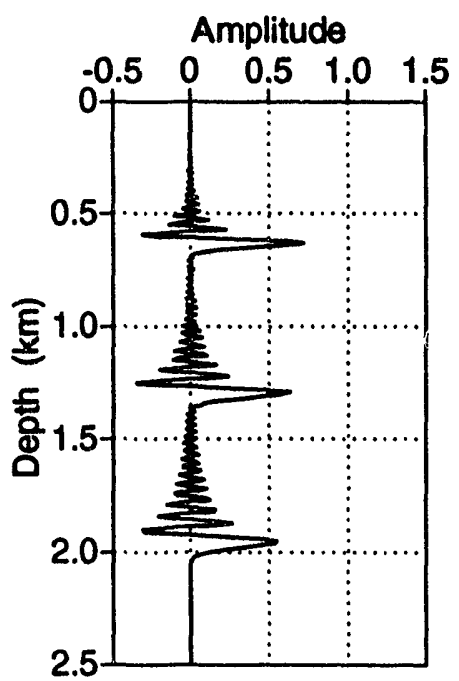

(b)

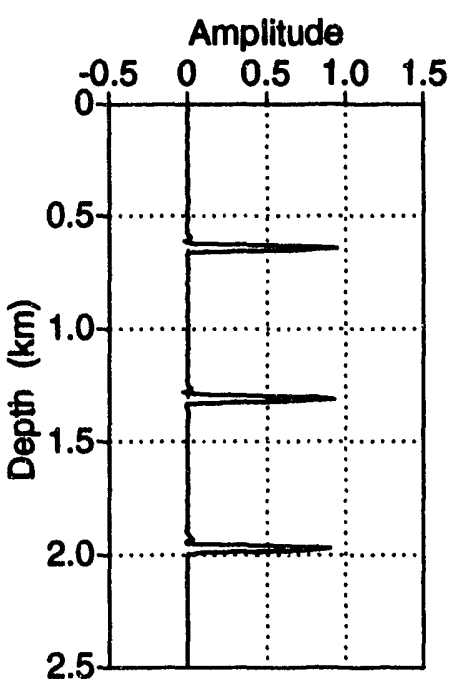

(c)

Fig. 4. One-dimensional test for isolated full-cycle of a sinusoid with a frequency of $40 \mathrm{~Hz}$ (five samples per wavelength), for a constant velocity of $2 \mathrm{~km} / \mathrm{s}$ and a constant density. (a) Ideal wavefield. (b) Wavefield obtained by a standard leapfrog finite-difference method. (c) Wavefield obtained with the FCT correction. 


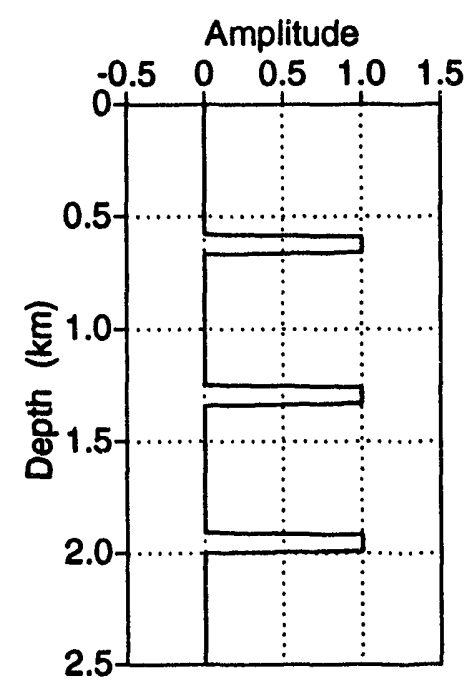

(a)

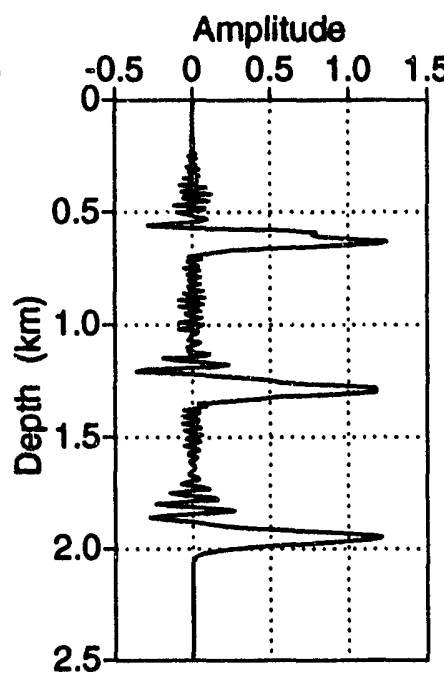

(b)

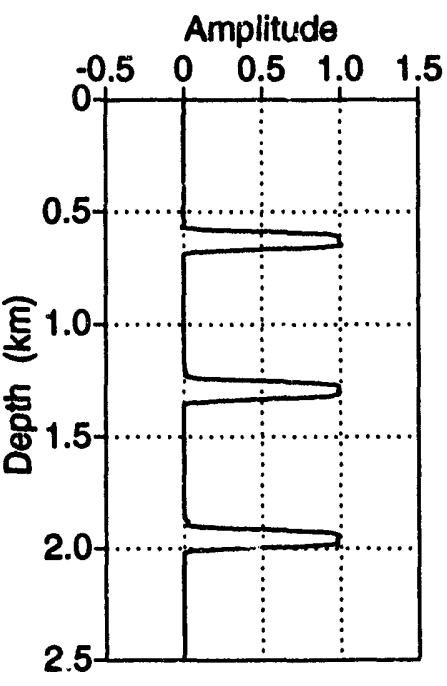

(c)

FIG. 5. One-dimensional test for isolated rectangle pulses (eight samples per pulse width), for a constant velocity of $2 \mathrm{~km} / \mathrm{s}$ and a constant density. (a) Ideal wavefield. (b) Wavefield obtained by standard leapfrog finite-üifference method. (c) Wavefield obtained with the FCT correction.

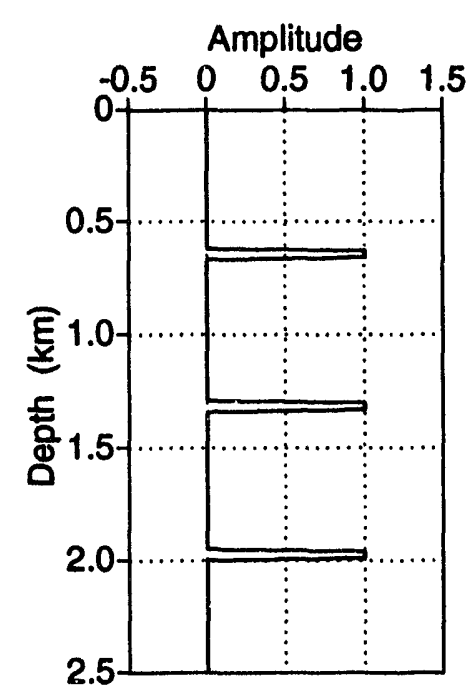

(a)

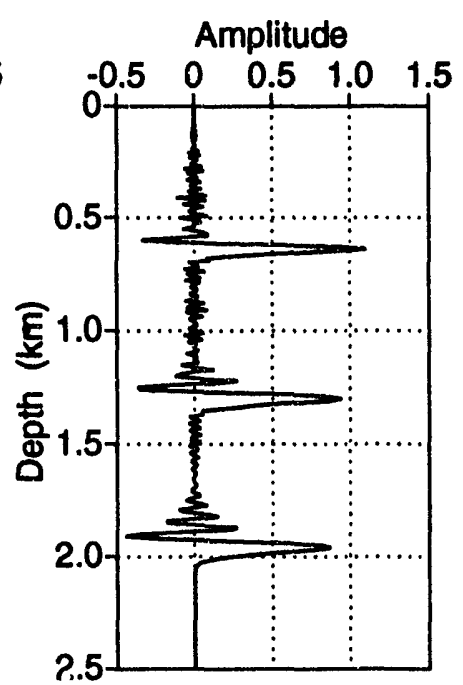

(b)

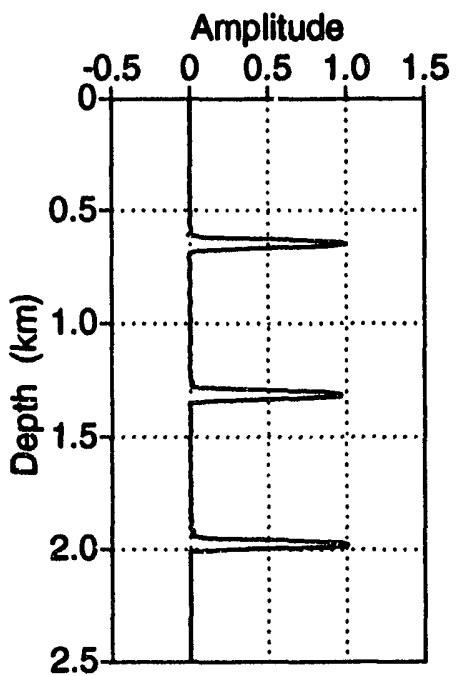

(c)

Fig. 6. One-dimensional test for isolated rectangle pulses (four samples per pulse width), for a constant velocicy of $2 \mathrm{~km} / \mathrm{s}$ and a constant density. (a) Ideal wavefield. (b) Wavefield obtained by standard leapfrog finite-difference method. (c) Wavefield obtained with the FCT correction. 


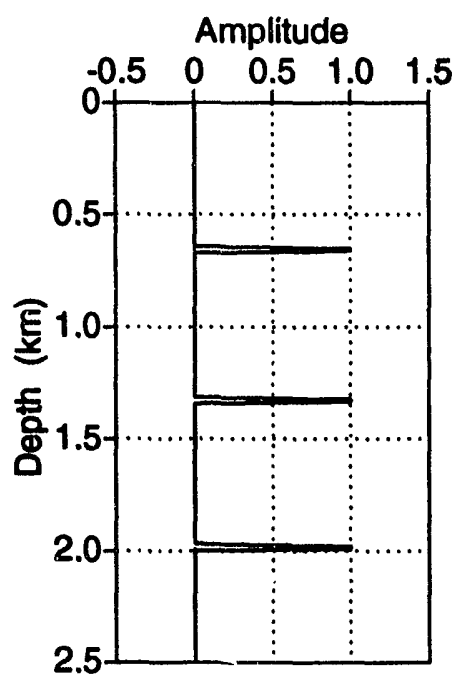

(a)

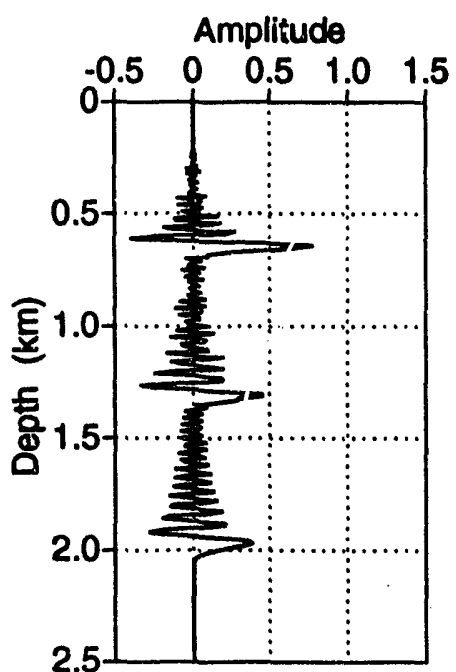

(b)

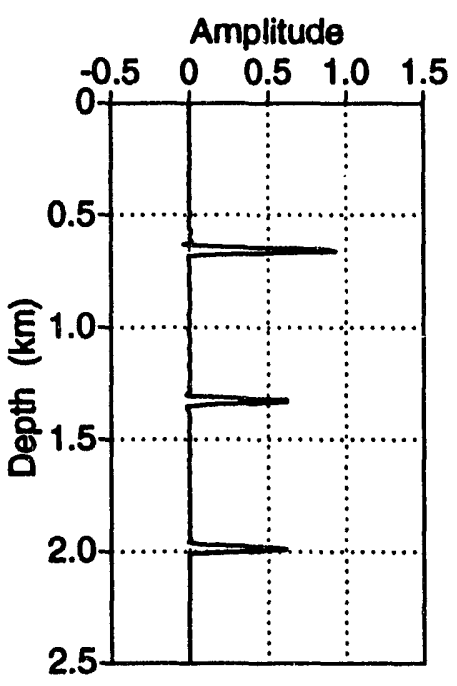

(c)

FIG. 7. One-dimensional test for isolated pulses (two samples per pulse width), for a constant velocity of $2 \mathrm{~km} / \mathrm{s}$ and a constant density. (a) Ideal wavefield. (b) Wavefield obtained by standard leapfrog finite-difference method. (c) Wavefield obtained with the FCT correction.

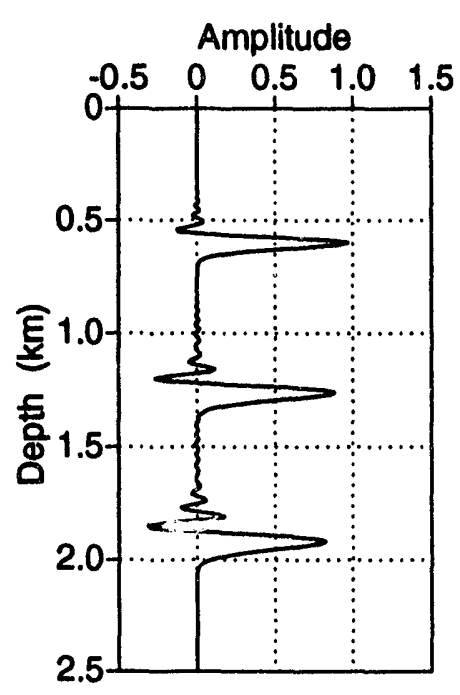

(a)

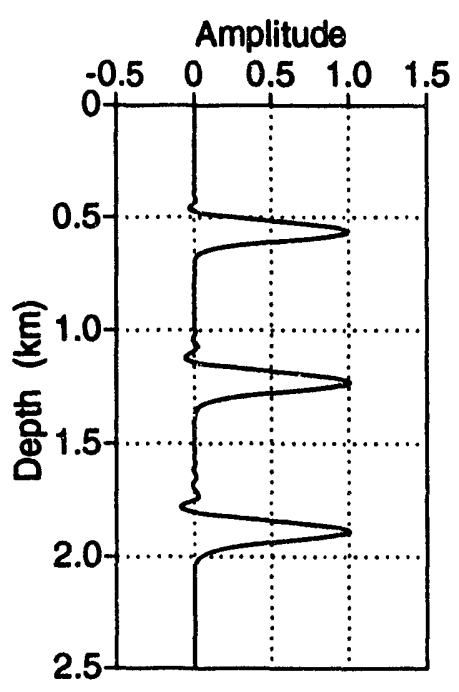

(b)

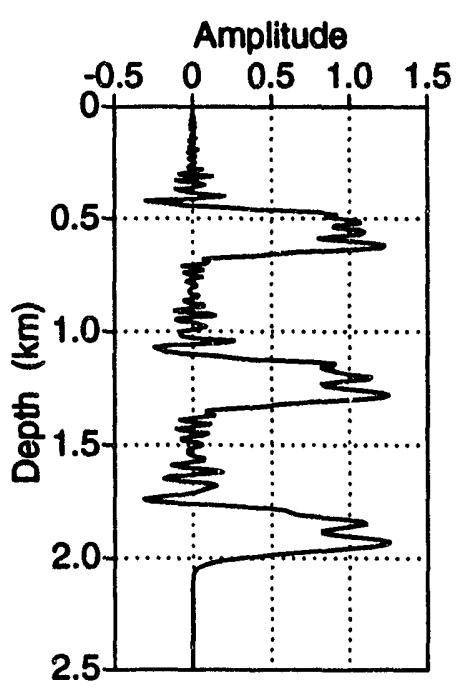

(c)

FIG. 8. One-dimensional tests using second-order finite-difference method for solving equation (1). (a) Wavefield for isolated sinusoid with eleven samples per wavelength. (b) Wavefield for isolated sinusoid with twenty samples per wavelength. (c) Wavefield for isolated pulses with twenty samples per pulse width. 


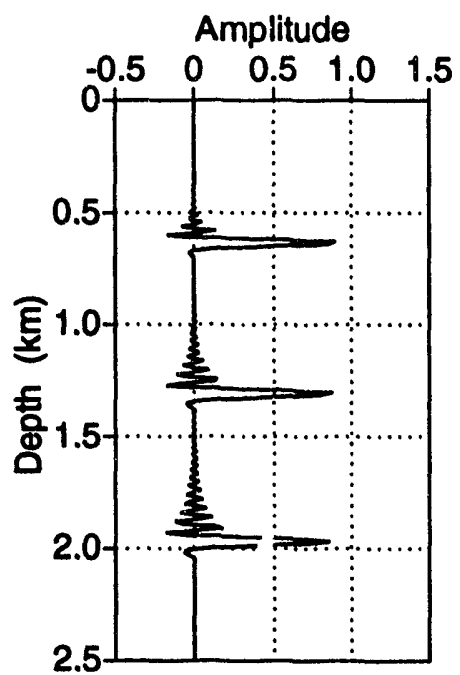

(a)

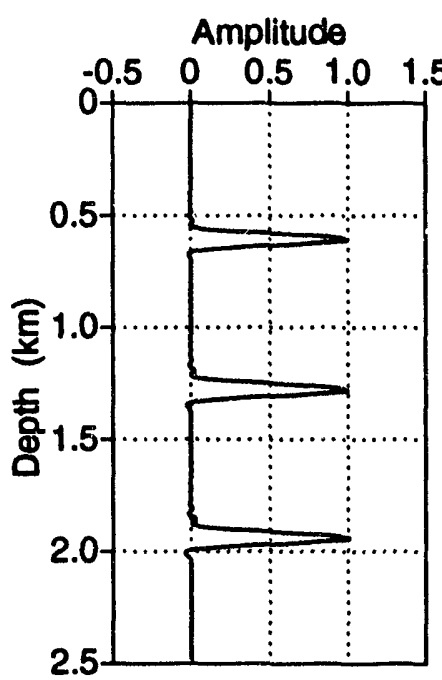

(b)

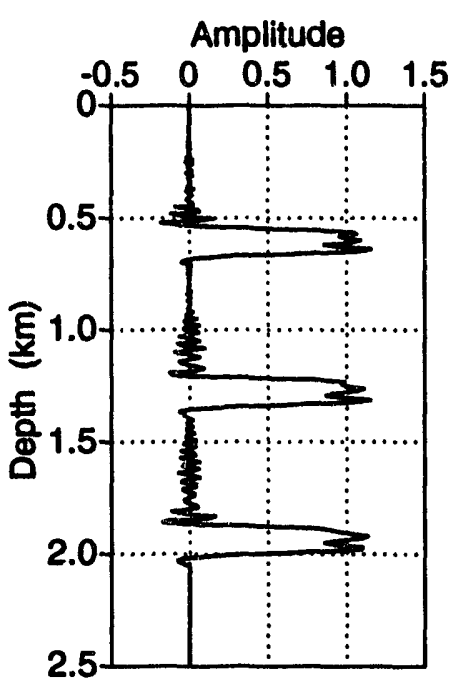

(c)

FIG. 9. One-dimensional tests using fourth-order finite-difference method for solving equation (1). (a) Wavefield for isolated sinusoid with six samples per wavelength. (b) Wavefield for isolated sinusoid with twelve samples per wavelength. (c) Wavefield for isolated pulses with twelve samples per pulse width.

order methods to the rectangle pulses, with sharp corners (Figures 8c and 9c), cannot give the correct wavefield - no matter how many samples per pulse width are used.

From these one-dimensional tests, we see that the FCT technique can treat large gradients and shocks without the usual dispersively generated ripples. The FCT algorithm not only removes the dispersive errors, but also yields a waveform with little distortion and only a few percent loss of amplitude if more than five samples per wavelength are used for the sinusoid and more than four samples per pulse width are used for the rectangular pulse. If too few samples per wavelength are used, the FCT method produces a mildly distorted waveform, but has significant loss of amplitude. For the narrowest pulse (two samples per pulse width), the amplitude loss, depending on propagation distance, is as great as about 35 percent in this test.

\section{Two-dimensional impulse responses}

Figure 10 shows the post-stack migration response of the FCT algorithm to three impulses (five samples per wavelength of the sinusoids) in 2.D space, where velocity is a constant $2.0 \mathrm{~km} / \mathrm{s}$. Here, density is again a constant, the spatial steps are $0.02 \mathrm{~km}(\Delta x=\Delta z=0.02 \mathrm{~km})$, and the time step $\Delta t$ is $2 \mathrm{~ms}$, running backward. The three clear semi-circular reflectors seen in the figure are in the correct positions, with no indication of numerical dispersion. Theory predicts that the migration impulse response in two dimensions carries a 45-degree phase shift (Claerbout, 1985), 
so a symmetrical input pulse generates a phase-distorted migration response. The migration impulse response in this test (Figure 10) has about 50-degree phase shift.

The theoretical amplitude variation with distance for 2-D impulse responses is rather complicated. As an approximation, the amplitude should be inversely proportional to the square-root of traveltime or distance (i.e. $\sqrt{t}$ or $\sqrt{r}$ ) for homogenous media (Bleistein 1984, 1986). In this test, the amplitude error increases witb traveling distance, for the first event the amplitude loss is about 3 percent, for the second event the amplitude loss is about 11 percent and for the third event the amplitude loss is about 15 percent.

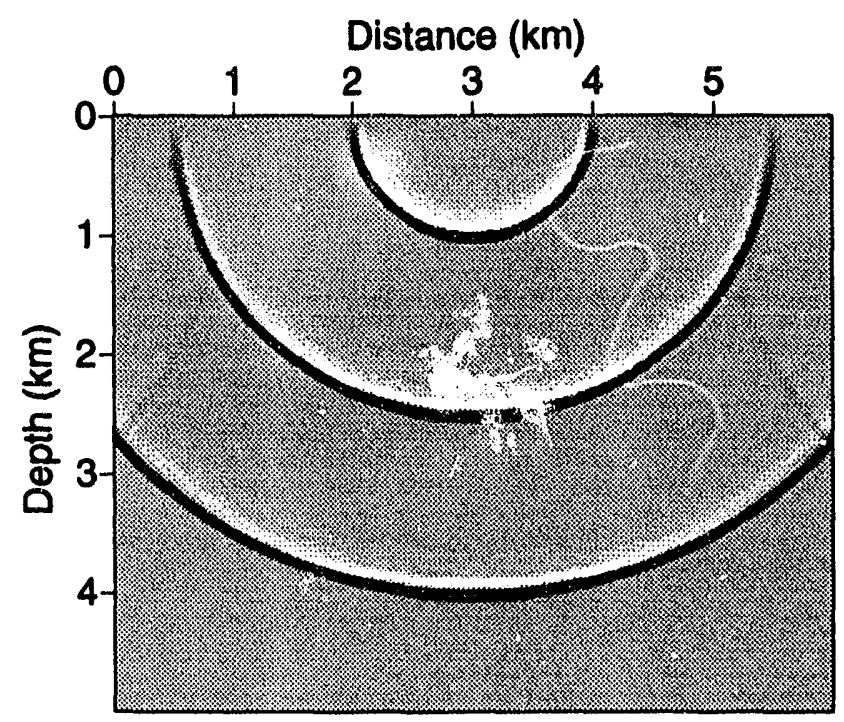

FIG. 10. Migration impulse responses by the flux-corrected transport method for a constant background velocity of $2.0 \mathrm{~km} / \mathrm{s}$ and constant density.

Figure 11 shows impulse responses (five samples per wavelength of the sinusoids) for velocity linearly increasing with depth, $c=2.0+0.6 z \mathrm{~km} / \mathrm{s}$, and with density kept constant. The spatial steps are $0.02 \mathrm{~km}(\Delta x=\Delta z=0.02 \mathrm{~km})$, and the time step $\Delta t$ is $1 \mathrm{~ms}$. Two events seen in the figure are in the right positions. The third event is out of the region of interest, but the result shows weak reflection of the third event due to the use of an imperfect absorbing boundary condition. For this velocity model, the FCT method can give good images for reflector dip beyond 90 degrees.

For lateral variable velocity, $c=1.6+0.2 x \mathrm{~km} / \mathrm{s}$, the post-stack migration response of the FCT algorithm to three impulses (five samples per wavelength of the sinusoids, and $\Delta t=2 \mathrm{~ms}$ ) is shown in Figure 12. Again, three clear semi-circular reflectors seen in the figure are in the correct positions, with no indication of numerical dispersion. 


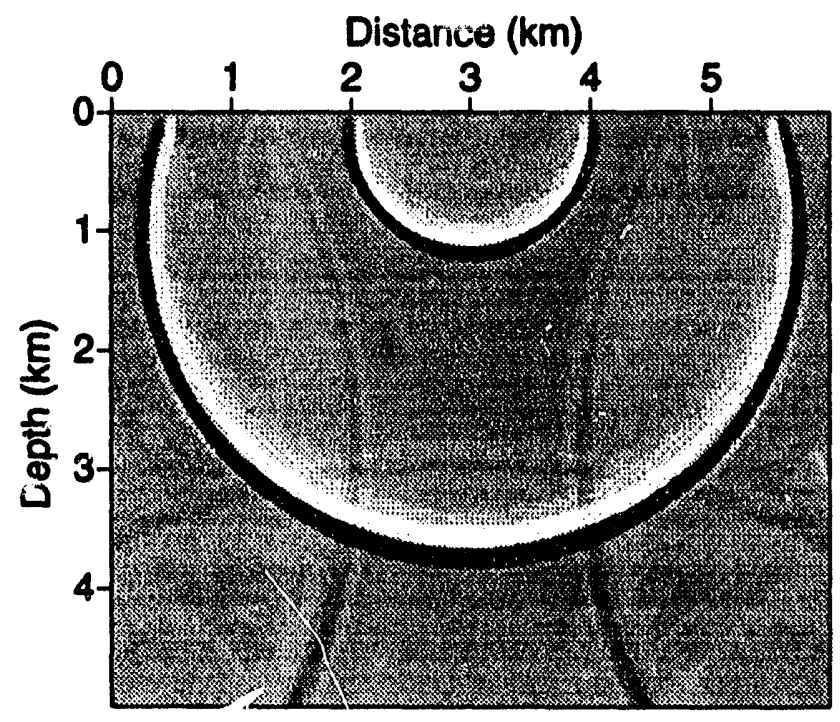

FIG. 11. Migration impulse response for velocity linearly increasing with depth, $c=2.0+0.6 z \mathrm{~km} / \mathrm{s}$, and constant density.

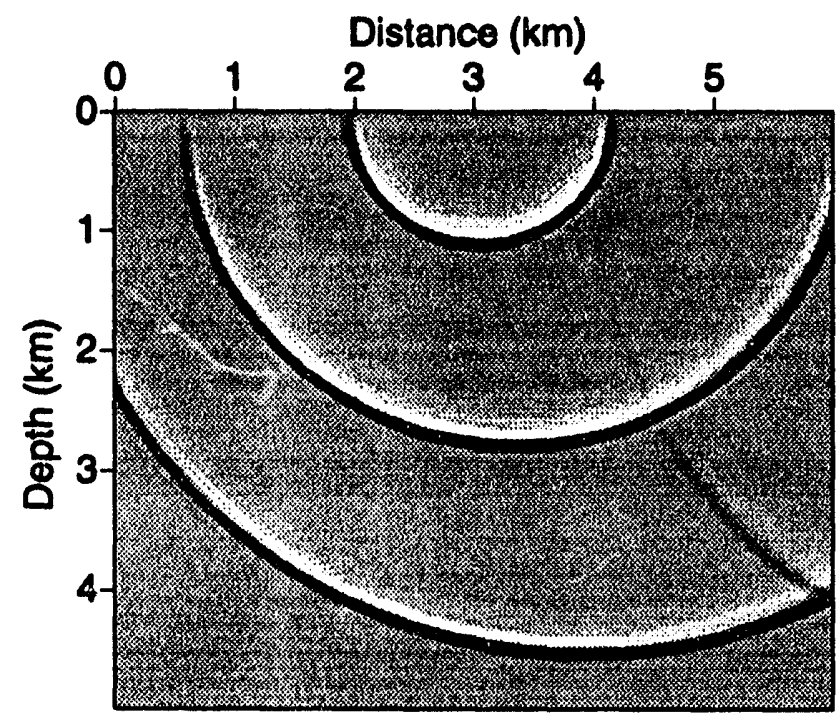

FIG. 12. Migration impulse response for velocity linearly increasing with distance, $c=1.6+0.2 x \mathrm{~km} / \mathrm{s}$, and constant density. 
Due to the lateral velocity variation, the centers of the semi-circular reflectors are shifted to the right.

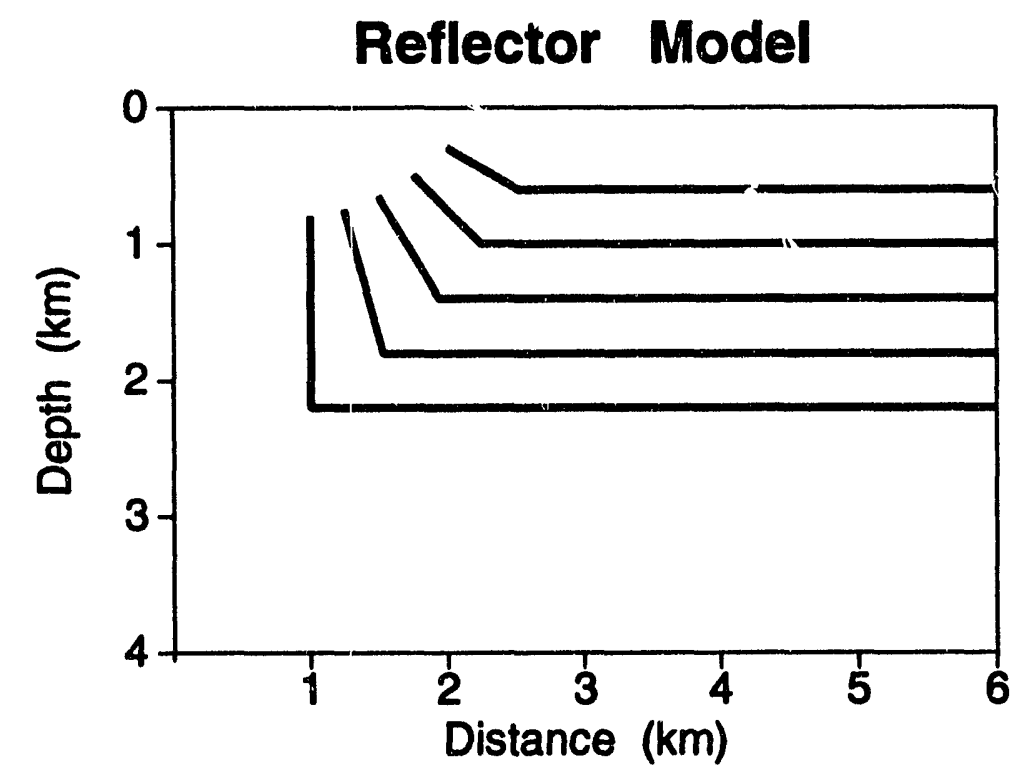

FIG. 13. Reflector model used to generate synthetic data for the tests described in Figures 15 through 18.

\section{Reflector model}

The model shown in Figure 13 has five reflectors, each with a horizontal segment and a segment with dip varying from 30 degrees to 90 degrees, in 15-degree increments. The irput zerc-offset time section for each of four different velocity models (Figure 14) was obtained by a Kirchhoff modeling program. The first of the fo:r models has constant velocity, and the other three have linear velocity variation, witi gradient in the vertical, horizontal, and intermediate directions, respectively. Figures 15 through 18 show the results of the FCT reverse-time depth migration applied to each of the zero-offset synthetic data sets in Figure 14. The spatial steps $\Delta x$ and $\Delta z$ in these tests are both $0.02 \mathrm{~km}$, and the time step $\Delta t$ is $2 \mathrm{~ms}$ for the cases of constant velocity and constant lateral velocity gradient, and is $1.5 \mathrm{~ms}$ for the other two cases. The input zero-offset synthetic data contain a symmetric Ricker wavelet with dominant frequency of $10 \mathrm{~Hz}$.

For the constant-velocity model (zero-offset synthetic data shown in Figure 14a), the migrated section (Figure 15) showis that a good image is obtained for horizontal reflectors and for reflectors with dip up to 60 degrees. For steeper reflectors, the image is poor because insufficient data were recorded. For a vertical reflector, synthetic data cannot be recorded in constant-velocity media, so no migrated image can be obtained. 


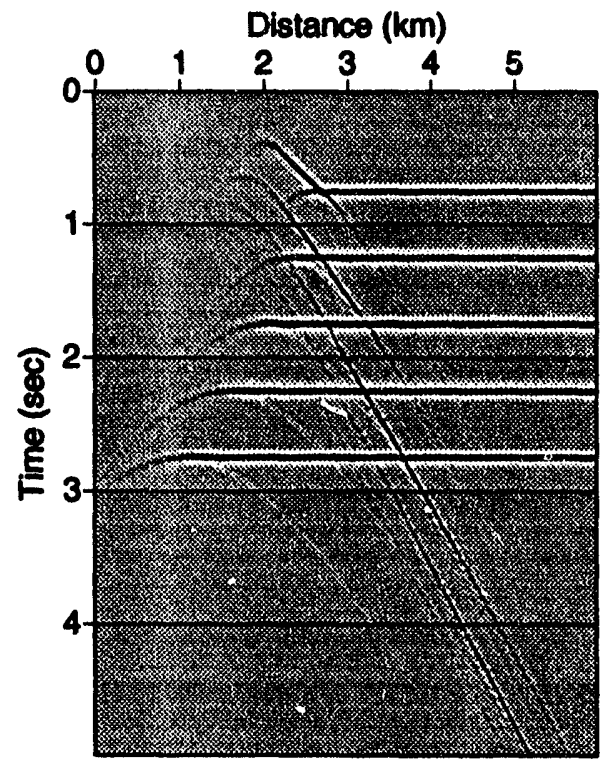

(a) $c=1.6 \mathrm{~km} / \mathrm{s}$

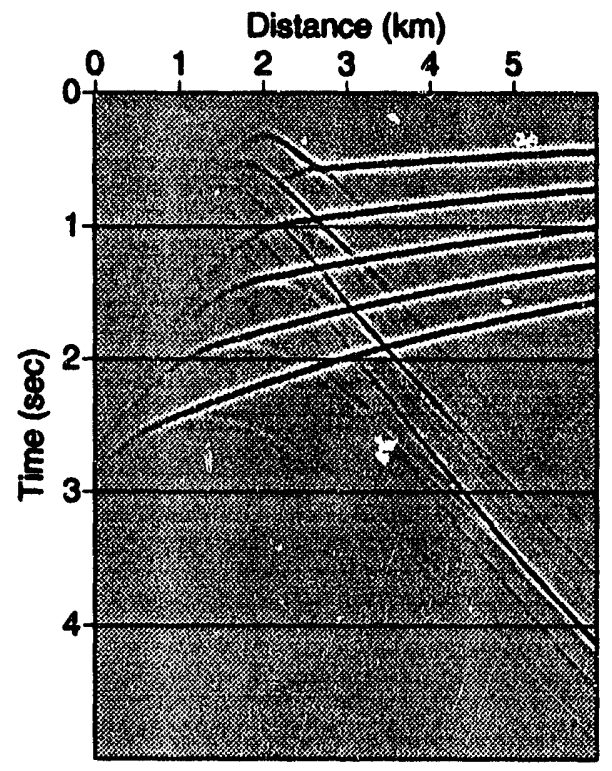

(c) $c(x)=1.6+0.2 x \mathrm{~km} / \mathrm{s}$

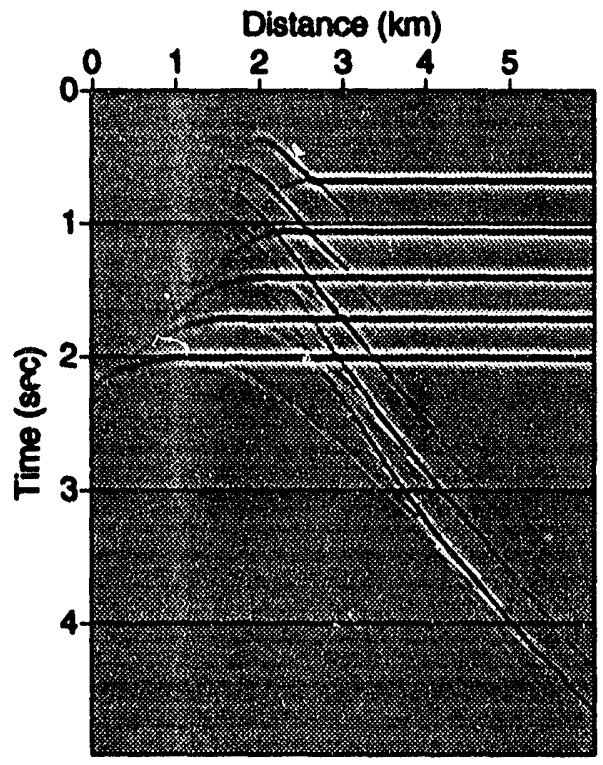

(b) $c(z)=1.6+0.6 z \mathrm{~km} / \mathrm{s}$

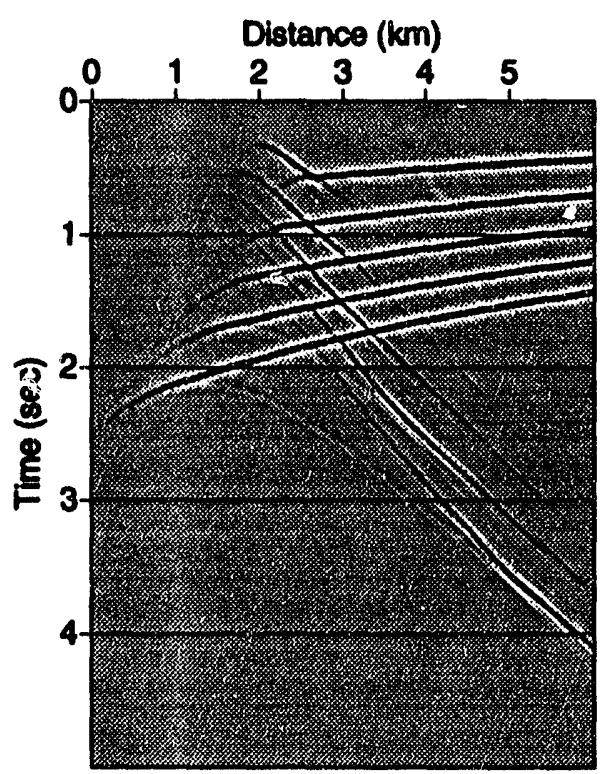

(d) $c(x, z)=1.5+0.2 x+0.35 z \mathrm{~km} / \mathrm{s}$

FIG. 14. Zero-offset synthetic time sections generated by Kirchhoff modeling for (a) constant velocity $c=1.6 \mathrm{~km} / \mathrm{s}$, (b) velocity linearly increasing with depth, (c) velocity linearly increasing in the horizontal direction, (d) velocity model with linear variation in $(x, z)$. 


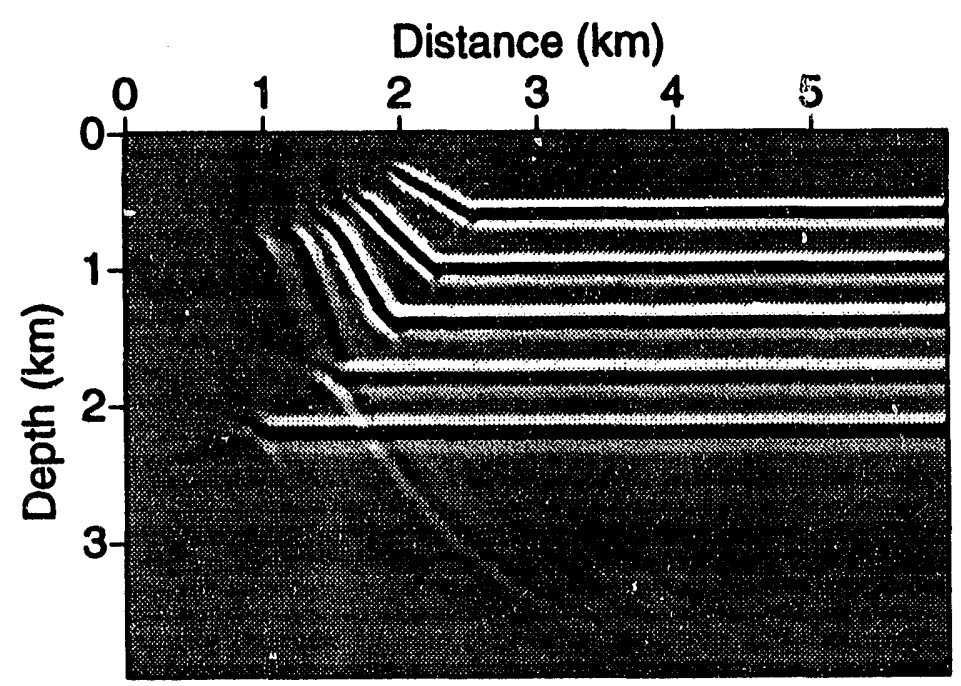

FIG. 15. Migrated section for constant velocity, $c=1.6 \mathrm{~km} / \mathrm{s}$, and constant density.

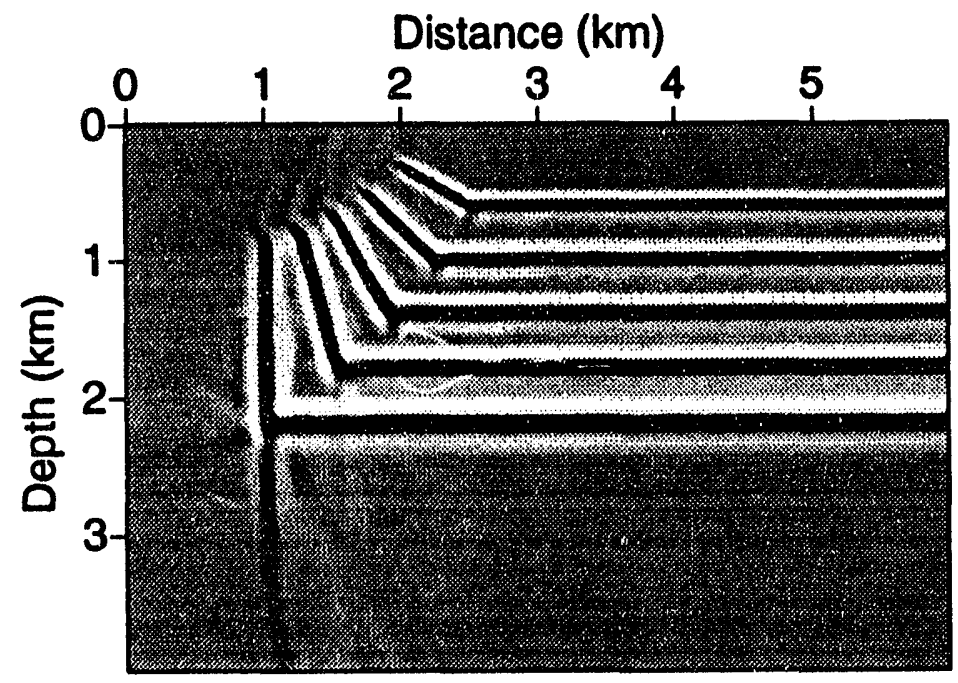

FIG. 16. Migrated section for velocity model $c(z)=1.6+0.6 z \mathrm{~km} / \mathrm{s}$, and constant density. 
Figure $14 \mathrm{~b}$ gives the synthetic data for velocity linearly increasing with depth, $c(z)=1.6+0.6 z \mathrm{~km} / \mathrm{s}$. The migrated section (Figure 16) indicates that the FCT method can accurately image the horizontal reflectors and dipping reflectors with all dips. The broadening of deeper events is caused by the increasing of velocity with depth, and the small artifacts at the joint and end of the reflectors are the result of insufficient synthetic data at the edges. These artifacts also are present in results obtained by a different migration method - the Kirchhoff integral method (Liu, 1992).

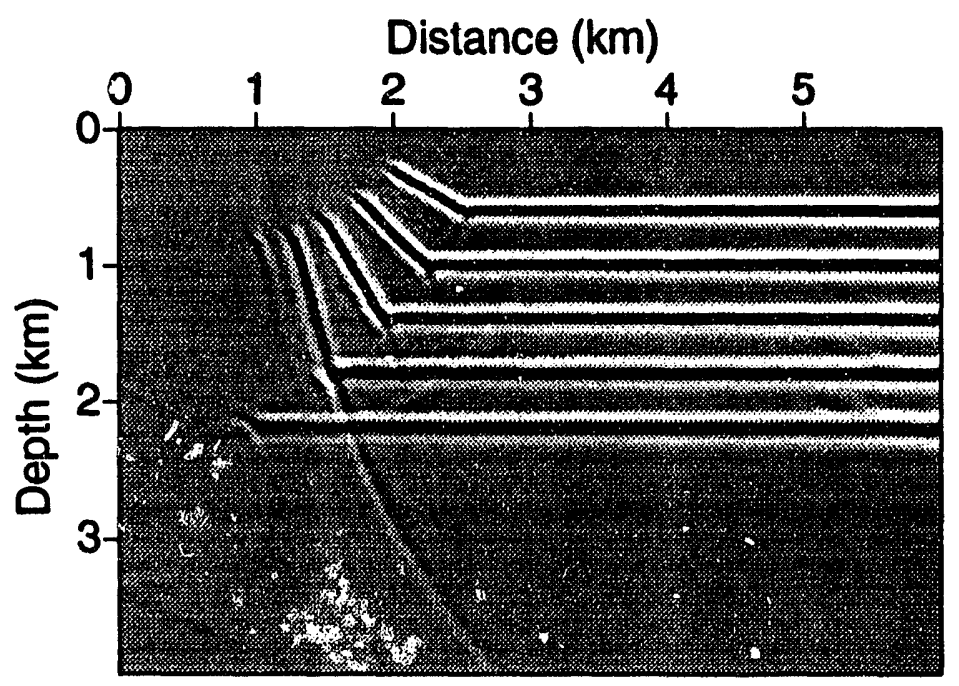

FIG. 17. Migrated section for velocity model $c(x)=1.6+0.2 x \mathrm{~km} / \mathrm{s}$, and constant density.

In the third model, velocity varies horizontally, $c(x)=1.6+0.2 x \mathrm{~km} / \mathrm{s}$. The migrated result (Figure 17) indicates that with laterally varying velocity, the FCT method can give clear and correct reflector positions for horizontal reflectors and for dipping reflectors with dip up to 75 degrees. The reflection from the 90 -degree reflector was not recorded within the data aperture (Figure 14c), so no image of it is generated. Note also in Figure 17 that amplitudes are weakened in the slow-velocity region. This weakening in amplitude also happens for migration by the Gaussian beam method with the same data set, suggesting that the weakening is related, in part, to a shortcoming in the data. However, the choice of coefficient $\eta_{1}$ and $\eta_{2}$ (see Appendix) in the FCT method also influences the amplitudes of the events. Here, I find the best results when the coefficient $\eta_{1}$ used in the FCT diffusion stage is linearly iucreasing toward the right, while the coefficient $\eta_{2}$ used in the FCT anti-diffusion stage is linearly decreasing in that direction. 


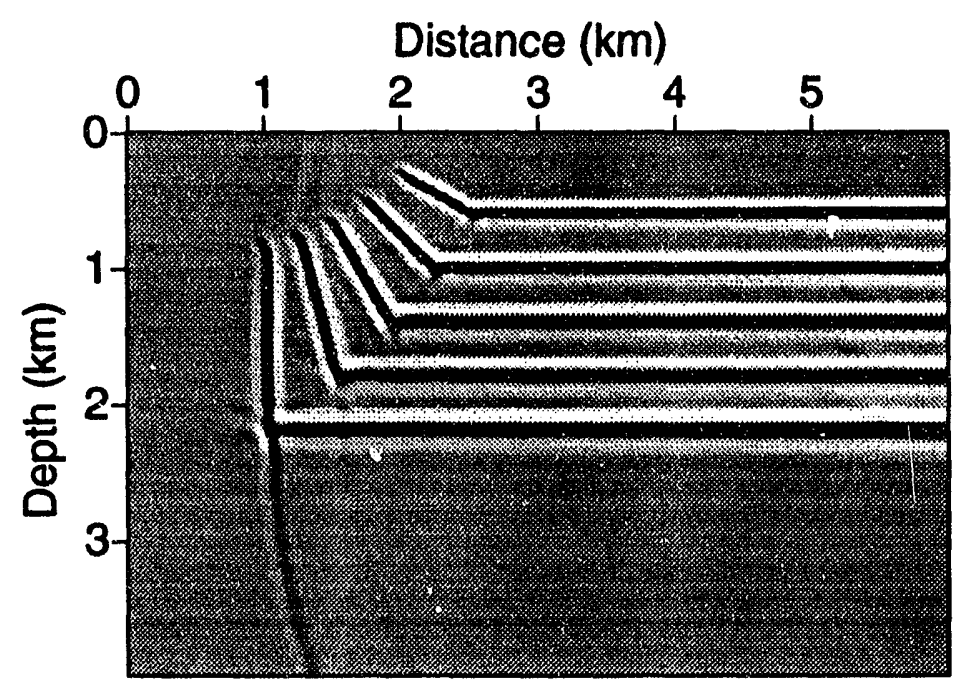

FIG. 18. Migrated section for velocity model $c(x, z)=1.5+0.2 x+0.35 z \mathrm{~km} / \mathrm{s}$, and constant density.

For a velocity field that is changing both vertically and horizontally, $c(x, z)=$ $1.5+0.2 x+0.35 z \mathrm{~km} / \mathrm{s}$, the migrated section (Figure 18) shows accurate positioning of the horizontal, dipping, and vertical reflectors. The result here is qualitatively somewhere between that for the two preceding cases. Again, the small artifacts at the joint and the end of the reflectors are the result of insufficient synthetic data at the edges.

\section{EFFICIENCY}

Comparisons of computational effort in the second-order FCT technique with that in conventional second-order and fourth-oi der finite-difference methods for seismic modeling and migration, suggests that the FCT method is more efficient.

For 2-D problems, the second-order conventional finite-difference method for solving equation (1) requires about $k N^{3}$ computations, here, assuming that the number of grid points in horizontal and depth directions, and the number of time steps are each equal to $N$. The second-order finite-difference method for solving equations (6) through (8) increases the amount of computation to about $1.6 \mathrm{kN} N^{3}$, which is nearly the same as that for the fourth-order finite-difference method for solving equation (1). The diffusion and anti-diffusion steps in the second-order FCT method again increase the computational effort, to about $4 k N^{3}$, but the FCT method gains in efficiency because fewer points per wavelength are required. In practice, twenty points per 
wavelength must be used for the second-order conventional finite-difference method and twelve points must be used for the fourth-order method to achieve acceptable accuracy. In contrast, for the FCT method, only five points need to be used to obtain comparable accuracy. Thus, as seen in Table 1, the FCT method should gain in speed by a factor of about sixteen compared with the conventional second-order finite-difference method, and by a factor of about five compared with the fourth-order method. For 3-D problems, the increased efficiency and speed of the FCT method should be even greater.

\begin{tabular}{|c|c|c|c|c|}
\hline Method & $\begin{array}{c}\text { Computational cost } \\
\text { proportional to }\end{array}$ & $\begin{array}{c}\text { Points } \\
\text { per wavelength }\end{array}$ & Time & Speed \\
\hline 2nd-order (1 eqn) & $k N^{3}$ & 20 & 1 & 1 \\
\hline 2nd-order (3 eqn) & $1.6 k N^{3}$ & 20 & 1.6 & 0.63 \\
\hline 4th-order (1 eqn) & $1.6 k N^{3}$ & 12 & 0.35 & 2.86 \\
\hline 2nd-order (3 eqn) FCT & $4 k N^{3}$ & 5 & 0.063 & 16 \\
\hline
\end{tabular}

Table 1. Comparison of relative computation effort and speed of the second-order FCT method with that of the second-order and the fourth-order conventional finitedifference methods for 2-D problems, and with second-order sulution of equations (6), (7) and (9). Here, the time and the speed of the second-order conventional finite-difference method for solving equation (1) are taken as unity.

\section{CONCLUSIONS}

Rewriting of the acoustic wave equation, gives a new set of the first-order partial differential equations, with no additional assumptions. These first-order equations are then amenable to solution by the flux-corrected transport method. The FCT finitedifference mod $\mathrm{gg}$ and reverse-time depth migration presented here can be applied to arbitrary velocity and density fields. The additional FCT steps require about 1.5 times more computation than that of the standard explicit finite-difference step. The FCT method, however, offers the opportunity to use a coarse grid (fewer samples per wavelength) to obtain accuracy in wave field extrapolation that is comparable to that obtained by conventional finite-difference methods. The FCT technique is still about triple the cost of the Kirchhoff integral method for 2-D applications. For 3-D migration and modeling, the computational effort of any finite-difference method (the FCT method in particular) is proportional to $N^{4}$, while that of the Kirchhoff integral method or Gaussian beam method is proportional to $N^{5}$, suggesting that the FCT method and the Kirchhoff integral method or Gaussian beam method might have competitive efficiency for 3-D problems.

The migrated results show that this method can produce good images of reflectors with dip beyond 90 degrees for velocity that increases with depth. Moreover, the FCT method can overcome problems of numerical dispersion that arise in standard 
non-diffusive, finite-difference algorithms where the wavefield has strong gradients or discontinuities.

\section{ACKNOWLEDGMENTS}

Thanks to Ken Larner and Jack Cohen for their critical reading of this paper. I also thank Dave Hale for helpful discussions and comments. I gratefully acknowledge the support by the members of the Consortium Project on Seismic Inverse Methods for Complex Structures at the Center for Wave Phenomena, Colorado School of Mines, and the support of the United States Department of Energy, grant number DE-FG0289ER14079. (This support does not constitute an endorsement by DOE of the views expressed in this paper.)

\section{REFERENCES}

Alford, R., Kelly, K., and Boore, D., 1974, Accuracy of finite-difference modeling of the acoustic wave equation: Geophysics, 39, 834-842.

Blacquiere, G., Debeye, H., Wapenaar, C., and Berkhout, A., 1989, 3D table-driven migration: Geophys. Prosp., З̃7, 925-958.

Bleistein, N., 1984, Mathematical methods for wave phenomena: Academic Press, Inc.

Bleistein, N., 1986, Two-and-one-half dimensional in-plane wave propagation: Geophys. Prosp., 34, 686-703.

Boris, J., and Book, D., 1973, Flux-corrected transport. I. SHASTA, A fluid transport algorithm that works: J. Comput. Phys., 11, 38-69.

Claerbout, J., 1985, Imaging of the earth's interior: Blackwell Scientific Publications, Inc.

Clayton, R., and Engquist, B., 1980, Absorbing side boundary conditions for wave equation migration: Geophysics, 45, 895-904.

Hale, D., and Witte, D., 1992, Migration: progress and predictions: Presented at the 62nd Ann. Internat. Mtg. Soc. Expl. Geophys., Expanded Abstracts, 610-612.

Kelly, K., Ward, R., Treitel, S., and Alford, R., 1976, Synthetic seismograms: a finite-difference approach: Geophysics, 41, 2-27.

Kosloff, D. and Baysal, E., 1983, Migration with the full acoustic wave equation: Geophysics, 48, 677-687.

Liu, Z., 1992, Seismic modeling and inversion for arbitrary velocity: CWP paper, Colorado School of Mines.

Reshef, M. and Kessler, D., 1989, Practical implementation of three-dimensional poststack depth migration: Geophysics, 54, 309-318. 


\section{APPENDIX: FLUX-CORRECTED TRANSPORT}

The flux-corrected transport (FCT) method (Boris and Book, 1973) was developed primarily for solving the first-order continuity equation in hydrodynamics. To apply the FCT method to seismic wavefield extrapolation, we need to solve equations (6) through (9). In this application, two-dimensional problems are considered. Without derivation, the FCT algorithm proceeds as follows.

1. Advance the solutions on a staggered grid by a standard finite-difference method (leapfrog algorithm has been applied here) and obtain $q_{i, j}^{n+1} u_{i+1 / 2, j}^{n+1}$ and $w_{i, j+1 / 2}^{n+1}$ at time level $n+1$. (For example, $q_{i, j}^{n}$ is the value of $q$ at time sample $n, x-$ coordinate sample $i$, and $z$-coordinate sample $j$ ). To save computation cost, the FCT correction (next few steps) need be performed only on the variable $q$. Such a correction is not necessary for computation of $u$ and $w$. Once $q$ is corrected, $u$ and $w$ can be computed directly from equations (7) and (9).

2. Compute diffusive fluxes at time level $n-1$ :

$$
\begin{array}{ll}
Q_{x_{i+1 / 2, j}}^{n-1}=\eta_{1}\left(q_{i+1, j}^{n-1}-q_{i, j}^{n-1}\right) & 0 \leq \eta_{\mathrm{i}} \leq 1 \\
Q_{z_{i, j+1 / 2}}^{n-1}=\eta_{1}\left(q_{i, j+1}^{n-1}-q_{i, j}^{n-1}\right) & 0 \leq \eta_{1} \leq 1,
\end{array}
$$

where $\eta_{1}$ is a coefficient that varies with position. In this application, $\eta_{1}$ is a linear function, with values between 0.02 and 0.1 . This function is empirically determined from a few numerical experiments by considering the amplitude treatment for horizontal events. Results are not critically sensitive to the choice of $\eta_{1}$ once it is close to a good value.

3. Compute diffusive fluxes at time level $n+1$, for the use in ste 6 below :

$$
\begin{array}{ll}
\tilde{Q}_{x i+1 / 2, j}^{n+1}=\eta_{2}\left(q_{i+1, j}^{n+1}-q_{i, j}^{n+1}\right) & 0 \leq \eta_{2} \leq 1 \\
\tilde{Q}_{z i, j+1 / 2}^{n+1}=\eta_{2}\left(q_{i, j+1}^{n+1}-q_{i, j}^{n+1}\right) & 0 \leq \eta_{2} \leq 1,
\end{array}
$$

with a similar choice for $\eta_{2}$ as for $\eta_{1}$ in step 2. The values or the function of $\eta_{2}$, however, may differ from that of $\eta_{1}$.

4. Modify (i.e. diffuse) the solution $q$ using $Q_{x}$ and $Q_{z}$; this process smooths the solution (also causes loss of amplitude) and eliminates the dispersive ripples :

$$
\begin{aligned}
\tilde{q}_{i, j}^{n+1}=q_{i, j}^{n+1} & +\left(Q_{x_{i+1 / 2, j}^{n-1}}^{n}-Q_{x_{i-1 / 2, j}^{n-1}}^{n-1}\right) \\
& +\left(Q_{z_{i, j+1 / 2}^{n-1}}-Q_{z_{i, j-1 / 2}^{n-1}}\right) .
\end{aligned}
$$

5. Take the differences of the diffused $\tilde{q}$ :

$$
\begin{aligned}
& X_{i+1 / 2, j}=\tilde{q}_{i+1, j}^{n+1}-\tilde{q}_{i, j}^{n+1} \\
& Z_{i, j+1 / 2}=\tilde{q}_{i, j+1}^{n+1}-\tilde{q}_{i, j}^{n+1} .
\end{aligned}
$$


6. Anti-diffuse the solution as follows, and obtain the corrected solution for $q$ :

$$
q_{i, j}^{n+1}=\tilde{q}_{i, j}^{n+1}-\left(X_{i+1 / 2, j}^{c}-X_{i-1 / 2, j}^{c}\right)-\left(Z_{i, j+1 / 2}^{c}-Z_{i, j-1 / 2}^{c}\right)
$$

where

$$
\begin{aligned}
& X_{i+1 / 2, j}^{c}=S_{x} \max \left\{0, \min \left[S_{x} X_{i-1 / 2, j}, \operatorname{abs}\left(\tilde{Q}_{x_{i+1 / 2, j}}^{n+1}\right), S_{x} X_{i+3 / 2, j}\right]\right\} \\
& Z_{i, j+1 / 2}^{c}=S_{z} \max \left\{0, \min \left[S_{z} Z_{i, j-1 / 2}, \operatorname{abs}\left(\tilde{Q}_{z i, j+1 / 2}^{n+1}\right), S_{z} Z_{i, j+3 / 2}\right]\right\} \\
& S_{x}=\operatorname{sign}\left\{\tilde{Q}_{x_{i+1 / 2, j}}^{n+1}\right\} \\
& S_{z}=\operatorname{sign}\left\{\tilde{Q}_{z i, j+1 / 2}^{n+1}\right\}
\end{aligned}
$$



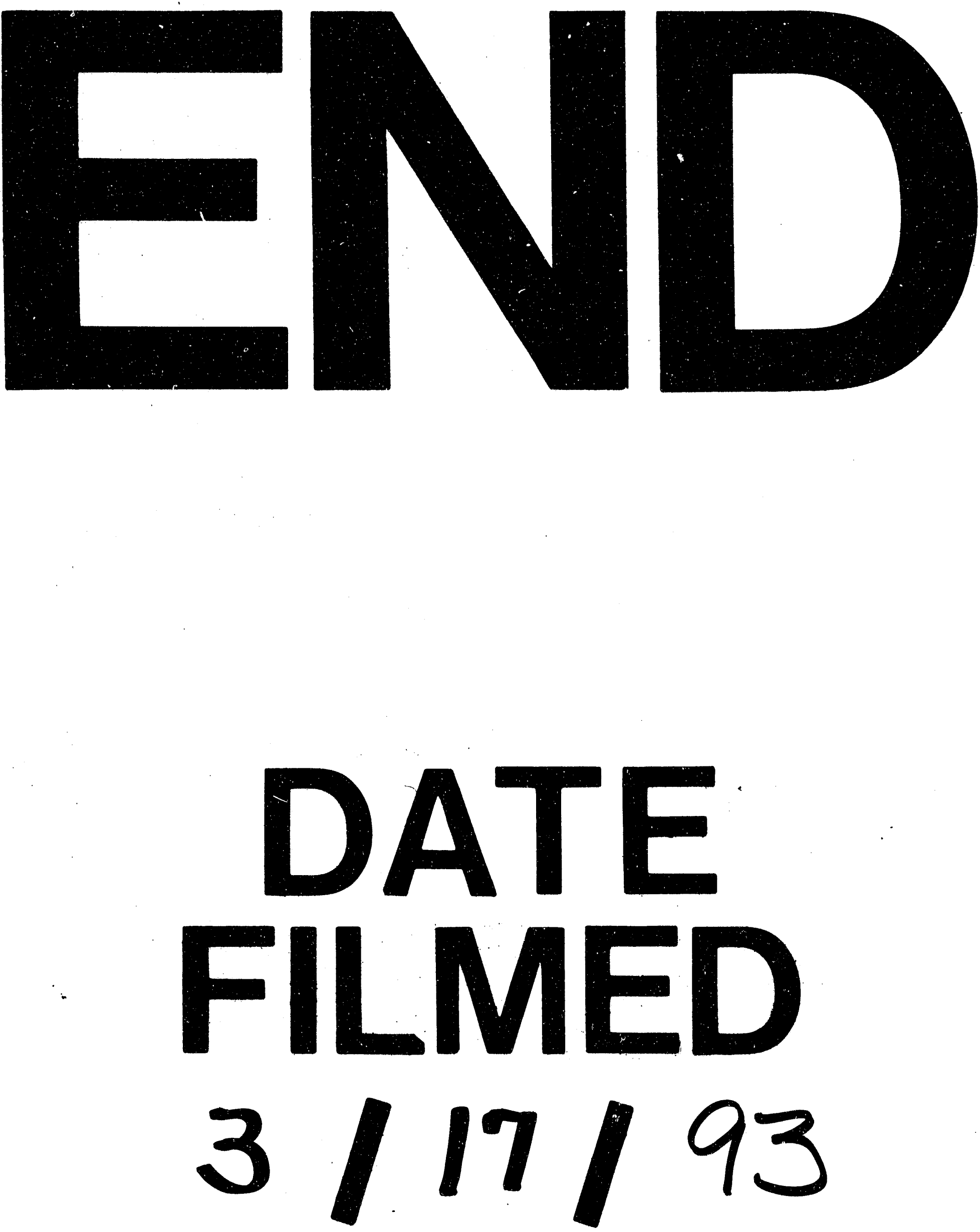

1 
